\title{
Adaptive FIR Filtering Using ABC Algorithm: a Noise Reduction Application on Mitral Valve Doppler Signal
}

\author{
Serdar Kockanat ${ }^{1}$, Turker Koza ${ }^{2}$, Nurhan Karaboga ${ }^{3}$, Ahmet Logoglu ${ }^{4}$ \\ ${ }^{1}$ Department of Electrical and Electronics Engineering, Faculty of Engineering, \\ Cumhuriyet University, \\ 58140, Sivas, Turkey \\ ${ }^{2}$ Department of Electronics, Vocational College, Bozok University, \\ 66200, Yozgat, Turkey \\ ${ }^{3}$ Department of Electrical and Electronics Engineering, Faculty of Engineering, Erciyes University, \\ 38039, Kayseri, Turkey \\ ${ }^{4}$ Department of Electronics and Automation Engineering, Vocational College, \\ Osmaniye Korkut Ata University, \\ 80000, Osmaniye, Turkey \\ skockanat@cumhuriyet.edu.tr
}

\begin{abstract}
The Doppler signal of mitral valve is a biomedical signals and it is acquired by Doppler ultrasound device from mitral valve of hearth. It contains useful information about mitral valve and it can be used to diagnose mitral valve diseases by doctors. However, biomedical signal might be contaminated with noise or disruptive interference. In literature, different approaches have been introduced to remove the noise or interference from biomedical signal, but adaptive filtering is most popular technique for noise filtering. Therefore, a novel adaptive noise cancellation techniques based FIR digital filter was proposed using artificial bee colony algorithm (ABC) and it is employed for the noise reduction on noisy mitral valve Doppler signal. So, the meaningful data about mitral valve was saved to diagnose mitral valve diseases using denoising approach. Then, this novel approach was compared with the other methods suggested in literature such as recursive least square (RLS) algorithm or discrete wavelet transform (DWT). Also, to demonstrate the efficiency, different digital filter order and noise cases were used suggested in literature.
\end{abstract}

Index Terms-Noise; Doppler signal; Optimization; ABC algorithm; Filter.

\section{INTRODUCTION}

Mitral valve diseases are diagnosed by different diagnosis techniques such as Doppler ultrasound, ECG (electrocardiography) or invasive techniques (e.g., angiography). The mitral valve has a major role for intracardiac blood flow. Therefore, the measurement of the intracardiac blood flow has a great importance with respect to early diagnosis of mitral valve diseases and it could be easily detected by the ultrasound technique. In medical practice, Doppler ultrasound is a popular, non-invasive and

Manuscript received 8 March, 2018; accepted 22 July, 2018.

This research was funded by a grant (No. FDK-2014-4859) from the Research Fund of Erciyes University. shortest way for assessing mitral valve diseases. Doppler ultrasound device uses high frequency sound allowing determination the speed and direction of the blood flow by utilizing the Doppler Effect and computes the blood flow velocity relating to Doppler-shift frequency and Doppler angle [1]. The Doppler signal is obtained by Doppler ultrasound device and analysed for diagnosing the mitral valve diseases. Conventionally, to analyse the Doppler signal, spectrogram is computed using STFT (short-time Fourier transform) and maximum frequency waveform is used to achieve indices [2]. Also, in literature, RI (resistance index) or PI (pulsatility index) are the most preferred spectrogram indices and waveforms which extracted from the Doppler signal. Especially, to diagnose mitral diseases, E and A waveforms are used. At the same time, a lot of studies that are based on the spectral estimation have been recently proposed for processing Doppler signal. During the Doppler signal acquisition in the electronic system, internal or external noise has a corruptive effect on both the Doppler signal and spectrogram depending on the extra undesired frequency component. Hence, in order to make an accurate diagnosis for mitral valve diseases, noise reduction in mitral valve Doppler signal is a key problem for further processing [3]. Also, in analysis and synthesis filter designs, mitral valve Doppler signal were successively reconstructed using QMF bank design based ABC algorithm in terms of phase delay and amplitude distortion [4].

In literature, one of the best ways for biomedical signal processing is adaptive filtering [5]-[7]. Because adaptive filtering has a great advantage compared with non-adaptive filtering. For example, the adaptive filter can be practically used for noise cancellation. In real time application, adaptive filter is very effective and preferred. Also, the filter coefficients can be self-adjusted by employing an 
optimization method controlled by a formulated error function. Therefore, the adaptive filtering is a trend study for researchers and practitioners in the area of signal processing [8], [9]. In design structure of adaptive filter, two main digital filters are used; FIR and IIR are called finite and infinite impulse response filters, respectively. But, the application of finite impulse response filter is more preferable than that of the infinite impulse response filter because of several advantages. The FIR filter is more stable and can be easily designed on most DSPs (digital signal processors) than IIR filter. Also, it has linear phase response and doesn't need any feedback. Moreover, the FIR filter is applied in widespread filter design approaches, such as quadrature mirror filter bank or two dimensional digital filter designs [10], [11].

Literature overview shows that various optimization algorithms have been used to solve the coefficient adjusting problem of the adaptive filter. Approximately, the employed algorithms are grouped in two categories; gradient based optimization algorithms and metaheuristic optimization algorithms. LMS (Least mean square), RLS (Recursive least square) and their hybrid algorithms are gradient based optimization algorithms. These algorithms have been successfully used in different application [12], [13]. On the other hand, DE (differential evolution), PSO (particle swarm optimization) and ABC (artificial bee colony) algorithm are more preferred metaheuristic algorithms for adaptive filter design approaches. Eberhart and Kennedy proposed the PSO algorithm in 1995 and now it is intensely used in adaptive filtering application of biomedical signals [14], [15]. Lately, the $\mathrm{ABC}$ algorithm is a robust and quick optimization method based intelligent foraging behaviour of honey bee swarm and in 2005, it was introduced by Karaboga [16]. In literature, the $\mathrm{ABC}$ algorithm has been widely used in various scientific disciplines and easily to solve real world optimization problems [17], [18]. The reason of the increasing interest in the $\mathrm{ABC}$ algorithm is that it is easy to implement, quite robust and convenient optimization algorithm [19], [20]. Especially, in recent years, the design problem of the adaptive filter has been examined using the $\mathrm{ABC}$ algorithm for biomedical signal processing [6], [7][21].

In the proposed work, a novel adaptive filtering approach based FIR filter using $\mathrm{ABC}$ algorithm is suggested to remove the undesired noise from the Doppler signal by using digital FIR filter. The results of proposed approach are compared with those of the gradient based LMS and RLS algorithms. Also, PSO, DE and conventional wavelet transform techniques were compared. The results show the difference in effectiveness and robustness between the novel adaptive filtering based $\mathrm{ABC}$ algorithm and other techniques for noise reduction process of the Doppler signal.

The proposed study is described as follows. Section II shows the proposed adaptive noise filtering approach. Section III describes the $\mathrm{ABC}$ algorithm step by step. In Section IV, the results of the ABC algorithm are presented and compared with those of the others techniques. Finally, Section $\mathrm{V}$ gives the conclusions.

\section{Proposed AdAptive Noise FILTERING ApPROACH}

ANC (Adaptive noise cancellation) was firstly addressed by Widrow and Glover in 1975 and they proposed a novel adaptive noise cancelling concept [22]. This concept is the most studied root solution for noise elimination problem [23], [24]. The ANC is a noise removing process and it can be realized in real-time processing application when the reference noise signal is obtained. Particularly, the ANC concept is successively utilized to remove noise on contaminated signal. Obtained meaningful signal can be used to diagnose the diseases by doctors and health care workers [6], [15], [25].

Figure 1 shows the proposed ANC approach. As depicted in Fig. 1, the signal $d(k)$ is the corrupted signal and it includes both the original signal $s(k)$ and the noise $n(k)$. The signal $x(k)$ is a reference noise signal and $n(k)$ is correlated with reference noise. $x(k)$ is applied by adaptive FIR filter to produce an estimate noise $y(k) . e(k)$ is the error signal and obtained by subtracting $y(k)$ from the $d(k)$. In Fig. 1, the input and output signal of the FIR digital filter is given as

$$
y(k)=\sum_{m=0}^{M-1} w_{m} x(k-m),
$$

where the input signal is $x(k)$, the output signal is $y(k), w_{m}$ is a vector that consists of filter coefficients, and $M$ specifies the order of the FIR digital filter. The filter coefficient vector can be presented as

$$
w=\left[b_{0} b_{1} \ldots b_{M-1}\right]
$$

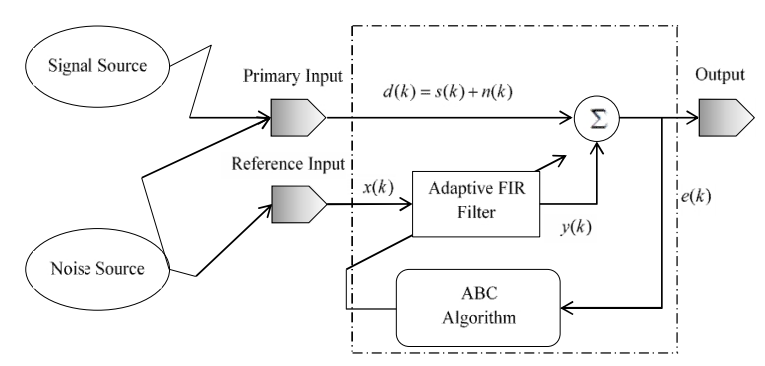

Fig. 1. The scheme of the proposed adaptive noise cancellation (ANC) approach.

In this study, the noise signal was chosen as AWGN (additive white Gaussian noise) and it is caused by random fluctuations in the signal. In the following equation, $e(k)$ is the error signal and fed back to the parameter adaptation algorithm. The coefficient vector of the adaptive FIR digital filter is adjusted by the parameter adaptation algorithm and minimization of the objective function is realized. The objective function, which is used in the parameter adaptation algorithm, is the mean square error (MSE) and represented as

$$
J(w)=\frac{1}{K} \sum_{k=1}^{K}[d(k)-y(k)]=E\left[|e(k)|^{2}\right],
$$

where $E$ represents the expected value.

In this work, the $\mathrm{ABC}$ algorithm was determined as major parameter adaptation algorithm and it optimized adaptively 
the filter coefficient vector of the FIR digital filter. So, the proposed ANC approach reduces the noise on the corrupted signal. In order to calculate the denoising performance, signal-to-noise ratio has been used and it is defined as

$$
\mathrm{SNR}=10 \log _{10} \frac{S}{N}(d B)
$$

where $S$ and $N$ represent the power of the original and noise signal.

\section{ABC ALGORITHM}

Recently, swarm based optimization algorithms have attracted much attention by scientists studying in optimization field and been used to solve different complicated problems in many different areas. Swarm intelligence algorithms simulate the behaviours of the swarms of animals, insects or bacteria. One of the swarm intelligence algorithms is artificial bee colony (ABC) algorithm. The ABC algorithm was firstly introduced by Karaboga in 2005 and it simulates the intelligent foraging behaviour of real honey bees for food sources [16]. Then, the performance and robustness of the $\mathrm{ABC}$ algorithm was compared with those of the other well-known metaheuristic algorithms [19], [20]. In the ABC algorithm, the behaviours of employed bees, onlookers and scout bees were analysed and their intelligent manners inspire to solving real world optimization problems.

In this paper, as the parameter adaptation algorithm, the $\mathrm{ABC}$ algorithm was employed and it optimizes adaptively the coefficients of the FIR digital filter. The designed FIR digital filter was adapted to improve the proposed adaptive noise cancellation approach. In this optimization process different from the other $\mathrm{ABC}$ based adaptive methods, there is no limitation using any window size or certain number of signal samples in adaptive filter design. Unlike, the whole mitral valve Doppler signal was iteratively processed by using adaptive FIR digital filter and the processing time is very short in terms of applicability for real time processing. The pseudo-code of the ABC algorithm applied to adaptive FIR filtering is given below:

Step 1: Initialization of the set (population) of solutions where each solution corresponds to a possible FIR digital filter: $x_{i j}, i=1, \ldots, S N, \mathrm{SN}$ is the number of solutions in the population. $j=1, \ldots, D, D$ represents the number of the coefficients of filter).

Step 2: Evaluation of the solutions corresponding to FIR filters by using (3).

Step 3: cycle $=1$.

Step 4: REPEAT.

Step 5: Generation of new solutions for employed bees by means of $v_{i j}=x_{i j}+\phi_{i j}\left(x_{i j}-x_{k j}\right)$ and evaluation of them by using (3), where $k \in(1, \ldots, S N), k \neq i$ and $j \in(1,2, \ldots, D)$ are randomly produced indexes, and $\phi_{i j}$ is randomly generated value between -1 and 1 .

Step 6: Implementation of greedy selection for solutions $x_{i}$ and $v_{i}$ found by the employed bees.
Step 7: Computation of probability values $p_{i}$ for the solutions (filters) by using the following functions $f i t_{i}(w)=\frac{1}{1+J_{i}(w)}$ and $p_{i}=\frac{f i t_{i}}{\sum_{n=1}^{S N} f i t_{n}}$, where fit $t_{i}$ and $J_{i}$ is the fitness and objective function values of solution $i$, respectively.

Step 8: Generation of new solutions $v_{i}$ for the onlooker bees by using $x_{i}$ chosen depending on its probability value $p_{i}$ and evaluation of $x_{i}$ and $v_{i}$ by (3).

Step 9: Use of greedy selection for the solutions (filters) determined by the onlooker bees.

Step 10: Determination of the exhausted solutions (filters) for the scout bees.

If there is any, generate new random solutions $x_{i}$ for it by using $x_{i}^{j}=x_{\min }^{j}+\operatorname{rand}[0,1]\left(x_{\max }^{j}-x_{\min }^{j}\right)$.

Step 11: Keeping in memory the best solution found so far.

Step 12: cycle $=$ cycle +1 .

Step 13: UNTIL cycle $=$ Maximum Cycle Number (MCN).

\section{RESUlts AND Discussions}

\section{A. Simulation and Data Acquisition Study}

In this work, the Doppler ultrasound signal of mitral valve was acquired by GE Vivid7 ultrasound device and sampling frequency was $44100 \mathrm{~Hz}$. Figure 2 shows the whole collected signal of mitral valve and randomly selected 250 samples with the certain range from 5000 to 5250 for this signal, respectively.

Additive white Gaussian noise (AWGN) is a corruptive noise on mitral valve Doppler signal and negatively affected to make an accurate diagnosis for mitral valve diseases. So, the effect of the AWGN on Doppler signal is an issue that should be examined. For this purpose, the noisy mitral valve Doppler signals with a SNR of $2 \mathrm{dBs}, 5 \mathrm{dBs}$ and $10 \mathrm{dBs}$ were produced by adding white Gaussian noise into the original Doppler signal of mitral valve. The SNR is a measure between original and noise signal and calculated by (4).

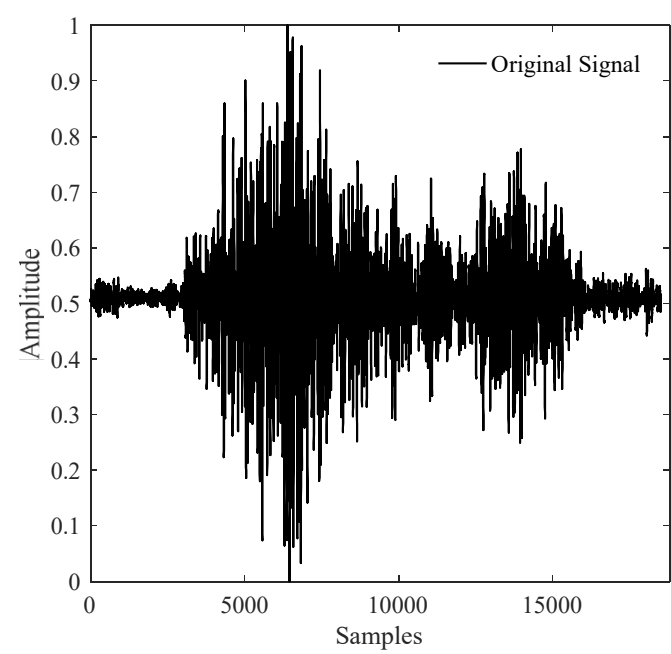

(a) 


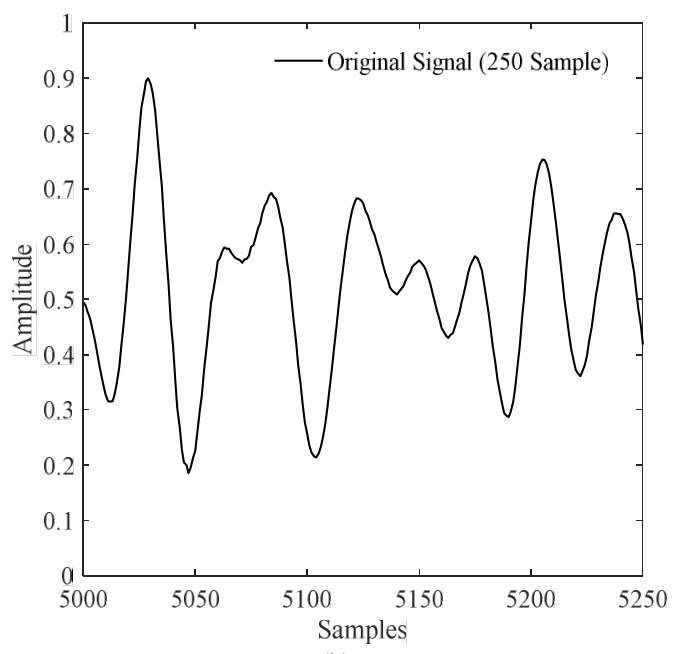

(b)

Fig. 2. Doppler signal of mitral valve: (a) for all samples; (b) for 250 samples with certain range from 5000 to 5250 .

Figure 3 shows the noisy Doppler signal of mitral valve with $2 \mathrm{~dB}$ and 250 samples with the certain range from 5000 to 5250 for both original and noisy signal, respectively. Also, in order to show the strength of the proposed ANC approach using $\mathrm{ABC}$ algorithm under worst noise conditions, the mitral valve Doppler signal were corrupted by varying AWGNs with highly SNR values.

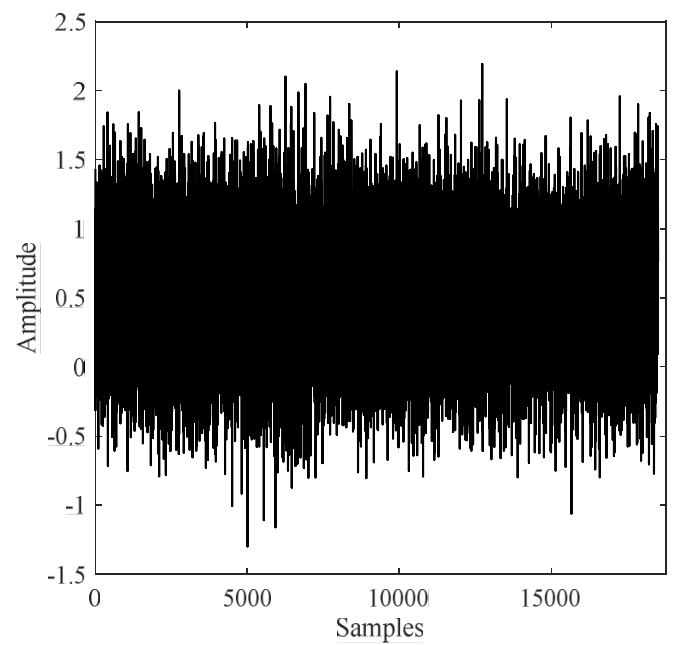

(a)

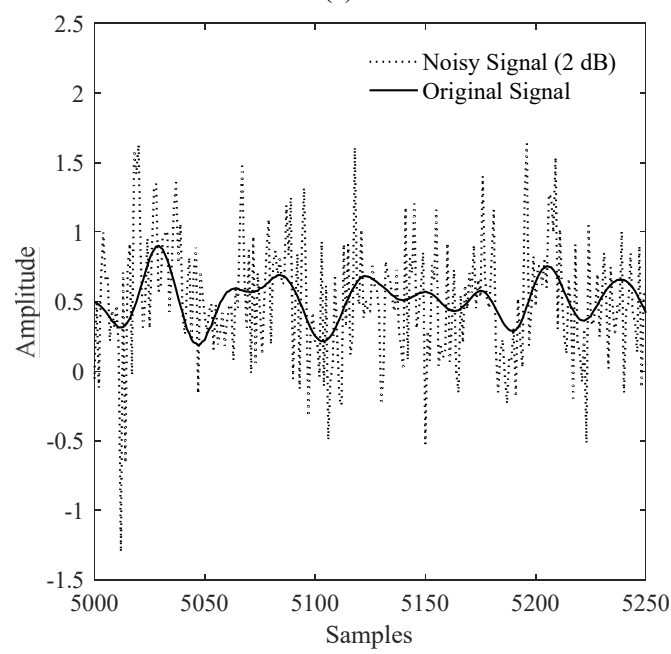

(b)

Fig. 3. Noisy mitral valve Doppler signal with $2 \mathrm{~dB}$ (a) for all samples (b) for 250 samples with certain range from 5000 to 5250 .
In this work, the $\mathrm{ABC}$ algorithm was proposed to remove noise on mitral valve Doppler signal of mitral valve employing proposed ANC approach with FIR digital filter. The control parameters of the $\mathrm{ABC}$ algorithm have a great importance on its performance and they were experimentally determined as recommend in literature [6]. Colony size, limit and maximum cycles were 20, 180 and 150, respectively. The algorithm was run 50 times with different random seeds and the initial values interval is selected as $[-1,1]$. The performance of the new $\mathrm{ABC}$ algorithm for the proposed ANC approach was compared with those of the LMS, RLS algorithms and the other methods in literature. For the best performance of the gradient based algorithms, LMS step size and RLS forgetting factor were experimentally selected 0.03 and 1 , respectively.

Also, the filter order is directly related to the computational complexity. If the coefficient size of the filter increases, more computation is needed. More computation causes long computational time for real time application. To examine the effect of varying filter order on the computational performance of the proposed ANC approach using $\mathrm{ABC}$ algorithm, a first, third and fifth order FIR filters were designed.

Unlike the other adaptive methods in literature, the new proposed ANC approach based $\mathrm{ABC}$ algorithm processed the whole mitral valve Doppler signal by shifting instead of the restricted signal using different windows size or limited samples.

\section{B. Performance of the ABC Algorithm for Adaptive Noise Cancellation Approach}

At first stage, the $\mathrm{ABC}$ algorithm was applied to realize the proposed ANC approach for denoising process. And then, in order to evaluate the performance of the $\mathrm{ABC}$ algorithm under worst condition, the FIR filter orders and SNR values were changed. Table 1 shows the obtained performance indicator values by using new proposed ANC approach based $\mathrm{ABC}$ algorithm for denoising process, such as filter order (F.O.), mean values (M.V.), standard deviation (S.D.) values, signal-to-noise rate (SNR, dB) and execution times (seconds).

TABLE I. THE RESULTS OBTAINED BY ABC ALGORITHM FOR

\begin{tabular}{|c|c|c|c|c|c|c|}
\hline $\begin{array}{c}\text { SNR } \\
\text { (dBs) } \\
\text { values } \\
\text { before } \\
\text { denoising }\end{array}$ & F.O. & M.V. & $\begin{array}{l}\text { S.D. } \\
\text { (e-6) }\end{array}$ & $\begin{array}{c}\text { Improved } \\
\text { SNR (dBs) } \\
\text { values } \\
\text { after } \\
\text { denoising }\end{array}$ & $\begin{array}{c}\text { Improved } \\
\text { SNR (\%) } \\
\text { after } \\
\text { denoising }\end{array}$ & $\begin{array}{c}\text { Time } \\
\text { (s) }\end{array}$ \\
\hline \multirow{3}{*}{2} & 1 & 0.2689 & 5.5193 & 38.10 & 1805 & 1.41 \\
\hline & 3 & 0.2689 & 10.678 & 36.10 & 1705 & 1.72 \\
\hline & 5 & 0.2689 & 15.359 & 34.75 & 1637 & 1.76 \\
\hline \multirow{3}{*}{5} & 1 & 0.2689 & 5.6510 & 38.41 & 668.25 & 1.01 \\
\hline & 3 & 0.2689 & 9.531 & 37.36 & 647.25 & 1.26 \\
\hline & 5 & 0.2689 & 14.523 & 35.60 & 612.06 & 1.42 \\
\hline \multirow{3}{*}{10} & 1 & 0.2689 & 5.5681 & 38.28 & 282.79 & 1.07 \\
\hline & 3 & 0.2689 & 10.182 & 37.02 & 270.25 & 1.25 \\
\hline & 5 & 0.2689 & 13.996 & 36.31 & 263.14 & 1.55 \\
\hline
\end{tabular}

In Table I, the denoising performance of the $\mathrm{ABC}$ algorithm was shown. Mean and standard values obtained by 
$\mathrm{ABC}$ algorithm for 50 runs with different random seeds are very small and it is good proof to demonstrate the robustness of the ABC algorithm. Improved SNR values are very high for first order FIR filters when the SNR values are changing ( $2 \mathrm{dBs}, 5 \mathrm{dBs}$ and $10 \mathrm{dBs}$ ) and they decreases by increasing the filter order. Also, the execution time decreases depending on the filter order. For instance, the execution times of the first order FIR filters are shorter than those of the third and fifth order filters for all SNR values. The coefficients of the designed first, third and fifth order FIR filters by using ABC algorithm are presented in Table II for SNR of $2 \mathrm{dBs}, 5 \mathrm{dBs}$ and $10 \mathrm{dBs}$.

TABLE II. COEFFICIENTS OBTAINED BY ABC ALGORITHM FOR SNR OF 2 DBS, 5 DBS AND 10 DBS.

\begin{tabular}{|c|c|c|c|c|c|c|c|}
\hline $\begin{array}{c}\text { SNR } \\
(\mathbf{d B})\end{array}$ & \multirow{2}{*}{ F.O. } & \multicolumn{7}{|c|}{ Coefficients } \\
\cline { 3 - 9 } & & $b_{0}$ & $b_{1}$ & $b_{2}$ & $b_{3}$ & $b_{4}$ & $b_{5}$ \\
\hline \multirow{3}{*}{2} & 1 & 0.9888 & -0.0109 & & & & \\
\hline & 3 & 0.9891 & -0.0084 & -0.0098 & -0.0100 & & \\
\hline & 5 & 0.9925 & -0.0125 & -0.0090 & -0.0091 & -0.0109 & -0.0054 \\
\hline \multirow{4}{*}{5} & 1 & 0.9848 & -0.0149 & & & & \\
\hline & 3 & 0.9843 & -0.0096 & -0.0094 & -0.0123 & & \\
\hline & 5 & 0.9849 & -0.0144 & -0.0096 & -0.0133 & -0.0085 & -0.0094 \\
\hline \multirow{3}{*}{10} & 1 & 0.9730 & -0.0274 & & & & \\
\cline { 2 - 8 } & 3 & 0.9748 & -0.0196 & -0.0187 & -0.0245 & & \\
\hline & 5 & 0.9744 & -0.0175 & -0.0197 & -0.0163 & -0.0243 & -0.0106 \\
\hline
\end{tabular}

Figure 4 shows the convergence graphics of mean square error of the first, third and fifth order FIR filters designed with the $\mathrm{ABC}$ algorithm for SNR of $2 \mathrm{~dB}$. From this figure, it is said that the convergence speeds depends on the filter order. As the filter order decreases, the convergence speed increases because the coefficient size to be optimized decreases and the design problem gets simpler. Also, the execution time is related to the convergence speed. As the convergence speed increases, the execution time decreases. Moreover, the results in Table I demonstrated that the execution time increases when the filter order increases.

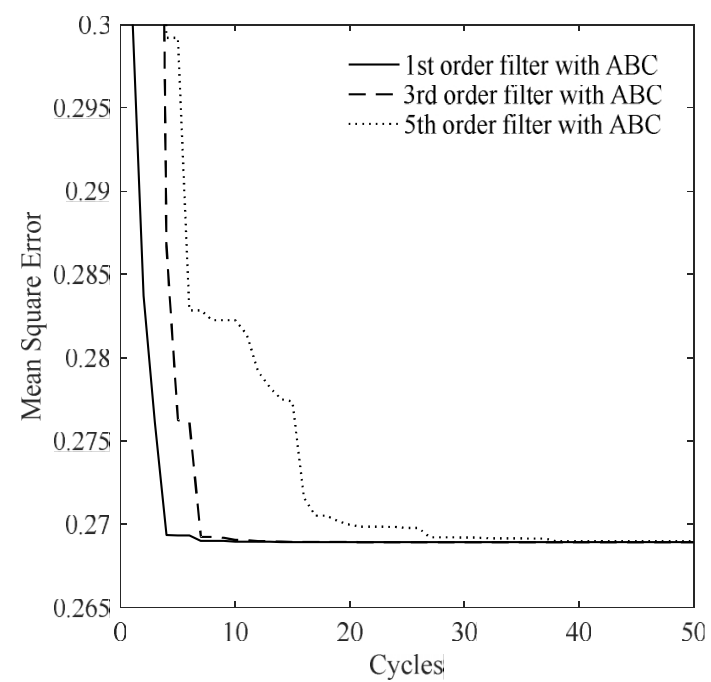

Fig. 4. The convergence graphics of the $\mathrm{ABC}$ algorithms with varying filter orders for SNR of $2 \mathrm{~dB}$.

Figure 5 shows the filtered 250 samples with certain range from 5000 to 5250 of mitral valve Doppler signal by using $\mathrm{ABC}$ algorithm with $1 \mathrm{st}, 3 \mathrm{rd}$ and 5 th order FIR filters for
SNR of $2 \mathrm{~dB}$. In here, instead of the whole mitral valve Doppler signal, only the limited samples are shown because it is difficult to see the difference between filtered and original signal for the whole Doppler signal.

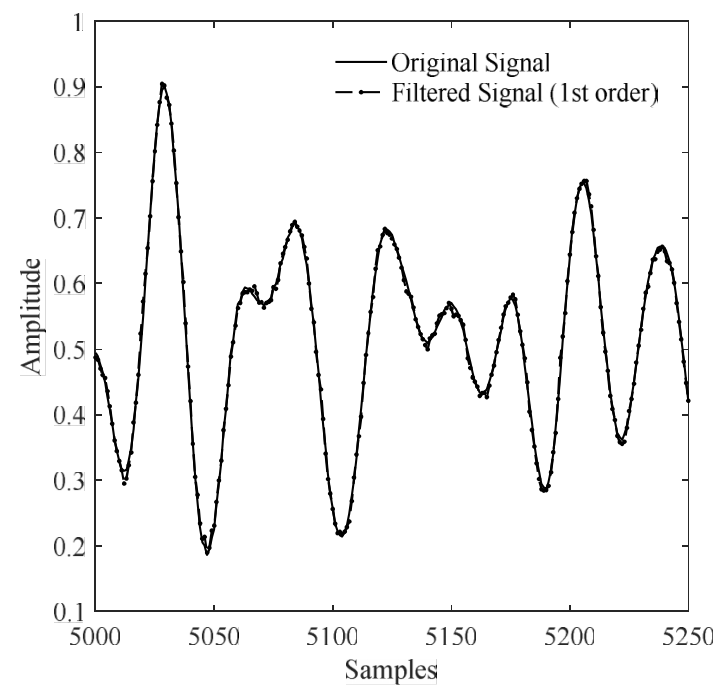

(a)

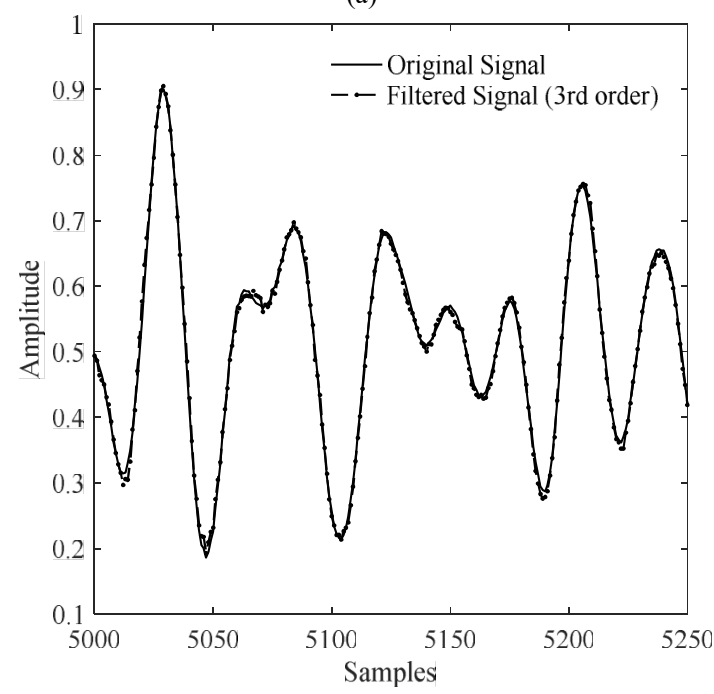

(b)

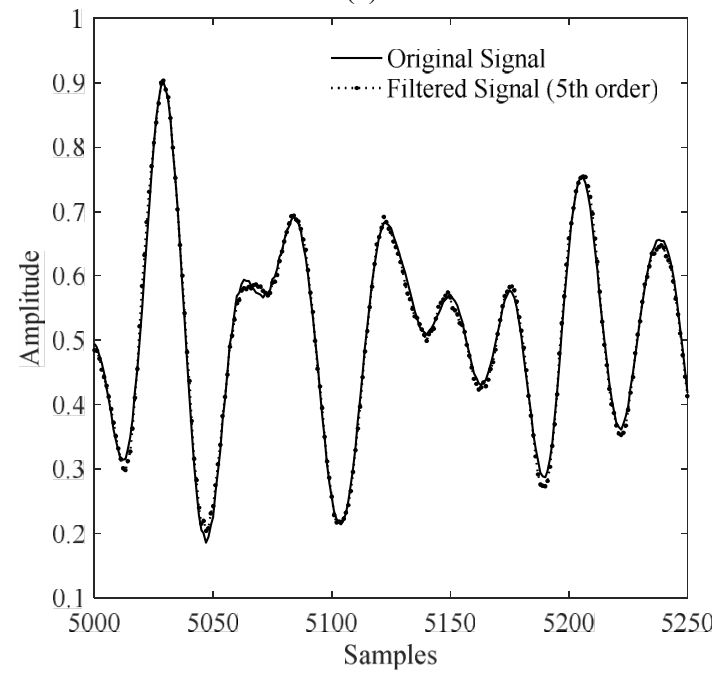

(c)

Fig. 5. Filtered signal by using $A B C$ algorithm with: (a) 1st, (b) 3rd and (c) 5 th order FIR filters for SNR of $2 \mathrm{~dB}$.

The denoising performance of the new proposed ANC approach using $\mathrm{ABC}$ algorithm for Doppler signal was compared with those of the LMS and RLS algorithms, 
metaheuristic algorithms based adaptive and non-adaptive structure and conventional methods based discrete wavelet approaches in literature [9]-[26]. Table III and Table IV show the SNR improvements of the Doppler signal using different methods with varying filter order and SNR values. For a fairly comparison, the results obtained by Zhang et al. [26] and Karaboga and Latifoglu [6] were taken from the literature and the proposed ANC approach was employed under same conditions recommend as in literature, such as same filter orders and same SNR values. From Table III and Table IV, it is concluded that the new proposed ANC approach using $\mathrm{ABC}$ algorithms shows better performance than the other methods for varying filter order and noisy cases ( $2 \mathrm{dBs}, 5 \mathrm{dBs}$ and $10 \mathrm{dBs})$. The RLS produces better results than the LMS algorithm in all cases. Unlike other adaptive and non-adaptive methods, the success of the new proposed ANC approach using $\mathrm{ABC}$ algorithm depends on the shifting usage of the whole Doppler signal in denoising process.

TABLE III. SNR IMPROVEMENTS FOR THE FIRST ORDER FILTER WITH VARYING SNR VALUES.

\begin{tabular}{|c|c|c|c|c|c|c|}
\hline \multicolumn{4}{|c|}{ SNR (dBs) values before denoising } & 2 & 5 & 10 \\
\hline \multirow{4}{*}{ 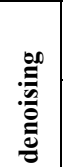 } & \multirow{2}{*}{\multicolumn{2}{|c|}{ Zhang et al. [26] }} & DWT & 182 & 55 & 16 \\
\hline & & & DWF & 265 & 94 & 39 \\
\hline & \multirow{6}{*}{$\begin{array}{c}\text { Karaboga } \\
\text { and } \\
\text { Latifoglu } \\
{[6]}\end{array}$} & \multirow{3}{*}{$\begin{array}{c}\text { Adaptive } \\
\text { filtering }\end{array}$} & $\mathrm{ABC}$ & 756 & 76 & 62 \\
\hline & & & PSO & 574 & 67 & 39 \\
\hline \multirow{7}{*}{ 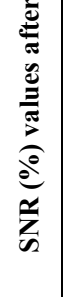 } & & & $\mathrm{DE}$ & 701 & 64 & 101 \\
\hline & & \multirow{3}{*}{$\begin{array}{l}\text { Non-adaptive } \\
\text { filtering }\end{array}$} & $\mathrm{ABC}$ & 1155 & 642 & 225 \\
\hline & & & PSO & 935 & 417 & 29 \\
\hline & & & $\mathrm{DE}$ & 929 & 414 & 28 \\
\hline & \multicolumn{3}{|c|}{ LMS } & 734.12 & 349.56 & 138.04 \\
\hline & \multicolumn{3}{|c|}{ RLS } & 1398 & 501.67 & 201.87 \\
\hline & \multicolumn{3}{|c|}{ Proposed } & 1805 & 668.25 & 282.79 \\
\hline
\end{tabular}

TABLE IV. THE SNR IMPROVEMENTS FOR THE FIFTH ORDER FILTER WITH VARYING SNR VALUES.

\begin{tabular}{|c|c|c|c|c|c|c|}
\hline \multicolumn{4}{|c|}{ SNR (dBs) values before denoising } & 2 & 5 & 10 \\
\hline \multirow{6}{*}{ 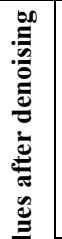 } & \multirow{6}{*}{$\begin{array}{c}\text { Karaboga } \\
\text { and } \\
\text { Latifoglu } \\
{[6]}\end{array}$} & \multirow{3}{*}{$\begin{array}{l}\text { Adaptive } \\
\text { filtering }\end{array}$} & $\mathrm{ABC}$ & 566 & 70 & 41 \\
\hline & & & PSO & 188 & 62 & 24 \\
\hline & & & $\mathrm{DE}$ & 442 & 58 & 48 \\
\hline & & \multirow{3}{*}{$\begin{array}{l}\text { Non- } \\
\text { adaptive } \\
\text { filtering }\end{array}$} & $\mathrm{ABC}$ & 1161 & 591 & 213 \\
\hline & & & PSO & 996 & 292 & 25 \\
\hline & & & $\mathrm{DE}$ & 467 & 176 & 21 \\
\hline$\sqrt{7}$ & \multicolumn{3}{|c|}{ LMS } & 774.95 & 293.10 & 124.43 \\
\hline อे & \multicolumn{3}{|c|}{ RLS } & 1165 & 407.16 & 154.49 \\
\hline$\frac{\mathscr{n}}{\sim}$ & \multicolumn{3}{|c|}{$\begin{array}{l}\text { Proposed adaptive } \\
\text { ABC filtering }\end{array}$} & 1637 & 612.06 & 263.14 \\
\hline
\end{tabular}

\section{CONCLUSIONS}

Mitral valve Doppler signal plays an important role to early diagnose mitral valve diseases and more meaningful and exact Doppler signal make easy the diagnostic procedure. So, noise reduction is a necessary process for the acquisition of Doppler signal of the mitral valve from the Doppler device. In this work, a new adaptive FIR filtering approach based ABC algorithm was successfully employed for noise cancellation. The first, third and fifth order FIR filter was used for denoising process under hard noise conditions, such as SNR of $2 \mathrm{dBs}, 5 \mathrm{dBs}$ and $10 \mathrm{dBs}$. The results indicated that the suggested ANC approach achieve
1805, 668.25 and 282.79 SNR (\%) improvements for $1^{\text {st }}$ order filter. Also, 1637, 612.06 and 263.14 SNR (\%) improvements were obtained for $5^{\text {th }}$ order filter. Also, the design of the FIR digital filter decreases the execution time of the denoising process.

\section{ACKNOWLEDGMENT}

The authors are grateful to reviewers for their valuable comments. Also, the authors thank to Turker Koza for collection of the mitral valve Doppler signal.

\section{REFERENCES}

[1] D. H. Evans, Doppler Ultrasound Physics, Instrumentation, and Clinical Application. Wiley, New York, 1989, pp. 270-302. DOI: 10.1002/bjs.1800770343.

[2] P. S. R. Diniz, Adaptive Filtering and Practical Implementations. Springer, USA, 2008, pp. 121-127. DOI: 10.1007/978-0-387-686066.

[3] B. Liu, Y. Wang, W. Wang, "Spectrogram enhancement algorithm: A soft thresholding-based approach", Ultrasound Med. Biol., vol. 25, pp. 839-846, 1999. DOI: 10.1016/S0301-5629(99)00024-1.

[4] T. Koza, N. Karaboga, "Quadrature mirror filter bank design for mitral valve doppler signal using artificial bee colony algorithm", Elektronika Ir Elektrotechnika, vol. 23, pp. 57-62, 2017. DOI: 10.5755/j01.eie.23.1.17584.

[5] N. Karaboga, F. Latifoglu, "Elimination of noise on transcranial Doppler signal using IIR filters designed with artificial bee colonyABC-algorithm”, Digital Signal Processing, vol. 23, pp. 1051-1058, 2013. DOI: $10.1016 /$ j.dsp.2012.09.015.

[6] N. Karaboga, F. Latifoglu, "Adaptive filtering transcranial Doppler signal by using artificial bee colony algorithm", Engineering Applications of Artificial Intelligence, vol. 26, pp. 677-684, 2013. DOI: 10.1016/j.engappai.2012.10.011.

[7] M. K. Ahirwal, A. Kumar, G. K. Singh, "Adaptive filtering of EEG/ERP through bounded range artificial bee colony (BR-ABC) algorithm”, Digital Signal Processing, vol. 25, pp. 164-172, 2014. DOI: $10.1016 /$ j.dsp.2013.10.019

[8] P. Upadhyay, R. Kar, D. Mandal, S. P. Ghoshal, V. Mukherjee, "A novel design method for optimal IIR system identification using opposition based harmony search algorithm", Journal of the Franklin Institute, vol. 351, no. 5, pp. 2454-2488, 2015. DOI: 10.1016/j.jfranklin.2014.01.001.

[9] L. Guohua, J. S. Brittain, P. Holland, J. Yianni, A. L. Green, J. F. Stein, T. Z. Aziz, S. Wang, "Removing ECG noise from surface EMG signals using adaptive filtering", Neuroscience Letters, vol. 462, no. 1, pp. 14-19, 2009. DOI: 10.1016/j.neulet.2009.06.063.

[10] J. Upendar, C. P. Gupta, G. K. Singh, "Designing of two channel quadrature mirror filter bank using particle swarm optimization", Digital Signal Processing, vol. 20, no. 10, pp. 304-313, 2010. DOI: 10.1016/j.dsp.2009.06.014.

[11] M. S. E Abadi, S. N. Aali, "The novel two-dimensional adaptive filter algorithms with the performance analysis", Signal Processing, vol. 103, pp. 348-366, 2014. DOI: 10.1016/j.sigpro.2013.12.016.

[12] T. F. Haddad, M. A. Khasawneh, "A new forced LMS-based adaptive algorithm utilizing the principle of potential energy", Journal of the Franklin Institute, vol. 337, no. 5, pp. 515-542, 2000. DOI: 10.1016/S0016-0032(00)00021-1.

[13] M. Almahamdy, H. B. Riley, "Performance study of different denoising methods for ECG signals", Procedia Computer Science, vol. 37, pp. 325-332, 2014. DOI: 10.1016/j.procs.2014.08.048.

[14] J. Kennedy, R. C. Eberhart, "Particle swarm optimization", in Proc. 4th IEEE Int. Conf. Neural Networks, 1995, pp. 1942-1948. DOI: 10.1109/ICNN.1995.488968.

[15] M. K. Ahirwal, A. Kumar, G. K. Singh, "Adaptive filtering of EEG/ERP through noise cancellers using an improved PSO algorithm", Swarm and Evolutionary Computations, vol. 14, pp. 7691, 2014. DOI: 10.1016/j.swevo. 2013.10.001.

[16] D. Karaboga, "An idea based on honey bee swarm for numerical optimization", Technical report-TR06, Erciyes University, Engineering Faculty, Computer Engineering Department, 2005. [Online]. Available: https://abc.erciyes.edu.tr/pub/tr06_2005.pdf

[17] D. Karaboga, B. Gorkemli, C. Ozturk, N. Karaboga, “A comprehensive survey: Artificial bee colony (ABC) algorithm and 
applications", Artificial Intelligence Review, vol. 42, no. 1, pp. 2157, 2014. DOI: 10.1007/s10462-012-9328-0.

[18] N. Karaboga, S. Kockanat, H. Dogan, "The parameter extraction of the thermally annealed Schottky barrier diode using the modified artificial bee colony", Applied Intelligence, vol. 38, no. 3, pp. 279288, 2013. DOI: 10.1007/s10489-012-0372-x.

[19] D. Karaboga, B. Basturk, "A powerful and efficient algorithm for numerical function optimization: Artificial bee colony $(\mathrm{ABC})$ algorithm", Journal Global Optimization, vol. 39, pp. 459-471, 2007. DOI: 10.1007/s10898-007-9149-x.

[20] D. Karaboga, B. Basturk, "On the performance of artificial bee colony (ABC) algorithm”, Applied Soft Computing, vol. 8, no. 1, pp. 687-697, 2008. DOI: 10.1016/j.asoc.2007.05.007.

[21] N. Karaboga, M. B. Cetinkaya, "A novel and efficient algorithm for adaptive filtering: artificial bee colony algorithm", Turkish Journal of Electrical Engineering \& Computer Sciences, vol. 19, no. 1, pp. 175-190, 2011. DOI: 10.3906/elk-0912-344.

[22] B. Widrow, J. R. Glover, “Adaptive noise cancelling: principles and applications", in Proc. IEEE 63, 1975, pp. 1692-1716. DOI: 10.1109/PROC.1975.10036.

[23] R. Bendoumia, M. Djendi, "Two-channel variable-step-size forwardand-backward adaptive algorithms for acoustic noise reduction and speech enhancement", Signal Processing, vol. 108, pp. 226-244, 2015. DOI: 10.1016/j.sigpro.2014.08.035.

[24] E. Zahedi, G. K. Beng, "Applicability of adaptive noise cancellation to fetal heart rate detection using photoplethysmography", Computers in Biology and Medicine, vol. 38, no. 1, pp. 31-41, 2008. DOI: 10.1016/j.compbiomed.2007.06.007.

[25] P. He, G. Wilson, C. Russell, "Removal of ocular artifacts from electroencephalogram by adaptive filtering", Medical and Biological Engineering and Computing, vol. 42, no. 3, pp. 407-412, 2004. DOI 10.1007/BF02344717.

[26] Y. Zhang, Y. Wang, W. Wang, B. Liu, "Doppler ultrasound signal denoising based on wavelet frames", IEEE Trans. Ultrason. Ferroelectr. Freq. Control, vol. 48, no. 3, pp. 709-716, 2001. DOI 10.1109/58.920698. 